\title{
Creating Quantum Emitters in Hexagonal Boron Nitride Deterministically on Chip-Compatible Substrates
}

\author{
Xiaohui $\mathrm{Xu}^{1}$, Zachariah O. Martin², Demid Sychev², Alexei S. Lagutchev ${ }^{2}$, Yong Chen ${ }^{2,3,4}$, Takashi \\ Taniguchi $^{5}$, Kenji Watanabe ${ }^{5}$, Vladimir M. Shalaev ${ }^{2}$, Alexandra Boltasseva ${ }^{1,2}$ \\ ${ }^{1}$ School of Materials Engineering, Purdue University, USA \\ ${ }^{2}$ School of Electrical and Computer Engineering, Purdue University, USA \\ ${ }^{3}$ Department of Physics and Astronomy, Purdue University, USA \\ ${ }^{4}$ Department of Physics and Astronomy, Aarhus University, Denmark \\ ${ }^{5}$ National Institute for Materials Science, Japan
}

\begin{abstract}
Two-dimensional hexagonal boron nitride $(\mathrm{hBN})$ that hosts bright room-temperature single-photon emitters (SPEs) is a promising material platform for quantum information applications. An important step towards the practical application of $\mathrm{hBN}$ is the on-demand, position-controlled generation of SPEs. Several strategies have been reported to achieve the deterministic creation of $\mathrm{hBN}$ SPEs. However, they either rely on a substrate nanopatterning procedure that is not compatible with integrated photonic devices or utilize a radiation source that might cause unpredictable damage to hBN and underlying substrates. Here, we report a radiationand lithography-free route to deterministically activate $\mathrm{hBN}$ SPEs by nanoindentation with an atomic force microscope (AFM) tip. The method is applied to thin hBN flakes (less than $25 \mathrm{~nm}$ in thickness) on flat silicon-dioxide-silicon substrates that can be readily integrated into on-chip photonic devices. The achieved SPEs yields are above $30 \%$ by utilizing multiple indent sizes, and a maximum yield of $36 \%$ is demonstrated for the indent size of around $400 \mathrm{~nm}$. Our results mark an important step towards the deterministic creation and integration of hBN SPEs with photonic and plasmonic on-chip devices.
\end{abstract}




\section{Introduction}

Solid-state single photon emitters (SPEs) are receiving increasing attention in the last decade due to their critical role in quantum information technologies including secure quantum communication, quantum photonic computing and quantum sensors. ${ }^{1-3}$ SPEs are typically composed of atomic defect structures in a solid-state host material that are suitable for integration with on-chip quantum photonic systems. Recently, two-dimensional (2D) van der Waals materials such as transition-metal dichalcogenides (TMDCs) ${ }^{4}$ and hexagonal boron nitride $(\mathrm{hBN})^{5}$ have been extensively investigated due to their capability to host SPEs. For instance, various types of SPEs operating at ambient conditions have been identified in $\mathrm{hBN}$, with emission ranging from ultraviolet (UV) to the near infra-red (NIR) spectral regime ${ }^{6,7}$. The atomic-scale thickness of 2D hBN not only enables high-efficiency light extraction, but also offers unparalleled advantages for integrating SPEs with plasmonic and photonic structures for hybrid quantum devices.

The practical integration of SPEs in 2D materials requires deterministic creation or activation of emitters at designated locations. Recent studies have reported a few methods to fabricate position-controlled SPEs in 2D materials based on strain engineering, ion/electron beam irradiation and controlled bottom-up growth. Strain/curvature-induced SPEs in hBN and TMDCs have been fabricated either by employing a nano-structured substrate (e.g., with nanopillars ${ }^{8}$, nanocones ${ }^{9}$, etc. ${ }^{10}$ ), or by deforming the $2 \mathrm{D}$ materials into a soft polymetric substrate ${ }^{11}$. However, both approaches have limited applications in quantum integrated photonics due to the involvement of patterned substrates or soft polymers. A similar strategy was utilized in a recent work to obtain SPEs that are not purely strain-induced in $\mathrm{hBN}$, by growing $\mathrm{hBN}$ on dielectric nanopillars using chemical vapor deposition (CVD) ${ }^{12}$. Aside from the above-mentioned methods, gallium focused ion beam (FIB) and electron beam have been demonstrated to create position-controlled SPEs in $\mathrm{hBN}$ flakes on a flat substrate ${ }^{13,14}$. However, the fluorescence contamination induced by highenergy gallium ion implantation could be a potential concern in practice, while SPE activation by electron beam suffers from limited spatial precision ( $>1 \mathrm{um}$ ).

In this work, we propose to deterministically activate room-temperature hBN SPEs by nano-indenting hBN with an AFM tip. The method is demonstrated for exfoliated, thin hBN flakes (less than $25 \mathrm{~nm}$ in thickness) placed on an unpatterned (flat) $\mathrm{SiO}_{2}$-coated silicon substrate. AFM probes with a diamond-like coating material are used to indent $\mathrm{hBN}$, without notable damage to the substrates. Our method utilizing an AFM tip is contamination-free, with no additional 
fluorescent contaminants introduced to $\mathrm{hBN}$, in contrast to radiation- or fabrication-based processes. ${ }^{8,12-14}$ By controlling the AFM probe displacement along the vertical direction, lateral indentation sizes ranging from $\sim 150 \mathrm{~nm}$ up to $800 \mathrm{~nm}$ are achieved with good repeatability. The nano-indentation is followed by argon annealing to fully activate SPEs at the indented sites. Our results provide a promising route to fabricate site-controlled hBN SPEs on chip-compatible substrates and pave the way to the realization of integrated quantum photonics with $\mathrm{hBN}$ SPEs. The capability to controllably pattern an array of quantum emitters also opens exciting possibilities such as realizing a "quantum mirror" ${ }^{15-17}$ and arrays of quantum photonic or hybrid sensors.

\section{Results and Discussion}

A schematic of the proposed nanoindentation technique utilizing AFM is shown in Figure 1a. First, hBN flakes exfoliated from high-quality $\mathrm{hBN}$ crystals are transferred to a $\mathrm{SiO}_{2}$-coated $\mathrm{Si}$ substrate. After solvent cleaning, thin flakes (less than $25 \mathrm{~nm}$ in thickness) are selected for further experiments using an optical microscope (see Figure 1b showing an AFM image of a typical hBN flake used for demonstrating the nanoindentation effect). Typically, flakes have thicknesses around 20-25 nm and roughness below $500 \mathrm{pm}$ (Figure 1), indicating an extremely clean and smooth surface. In the AFM contact mode, the cantilever coated with diamond-like carbon is brought into contact with hBN and then gets indented into the flake with further cantilever displacement along the vertical direction. To ensure effective indentation, the cantilever is held at its maximum displaced position for 2 seconds before retraction. By adjusting the maximum cantilever displacement $\left(\Delta z_{\max }\right)$, indents with varying lateral sizes could be obtained, as shown in Figure 1c. To explore the indent structure, we scanned one of the indents (Figure 1c) and extracted a line profile across the indented area. As illustrated in Figure 1d, the area is composed of a dip created in $\mathrm{hBN}$ as well as two bent $\mathrm{hBN}$ pieces resulting from the AFM probe impact. To demonstrate how the lateral size of indented dimples in hBN scales with the cantilever displacement, we measured all the indent sizes as a function of $\Delta z_{\max }$ (Figure 1c and Figure 1e). The method used to determine lateral indent sizes in this work could be found in Section I, Supplementary Information. The lateral indent size scales almost linearly with $\Delta z_{\max }$ in the tested $\Delta z_{\max }$ range. We also note that changing $\Delta z_{\max }$ mainly affects the lateral dimension, rather than the depth of resulting indents. The indentation typically creates dimples with depths less than or comparable to the hBN flake thickness even for the largest indents tested (Figure S2, Supplementary Information). This implies 
that the developed indentation procedure "pokes" hBN without deforming or damaging the substrate.
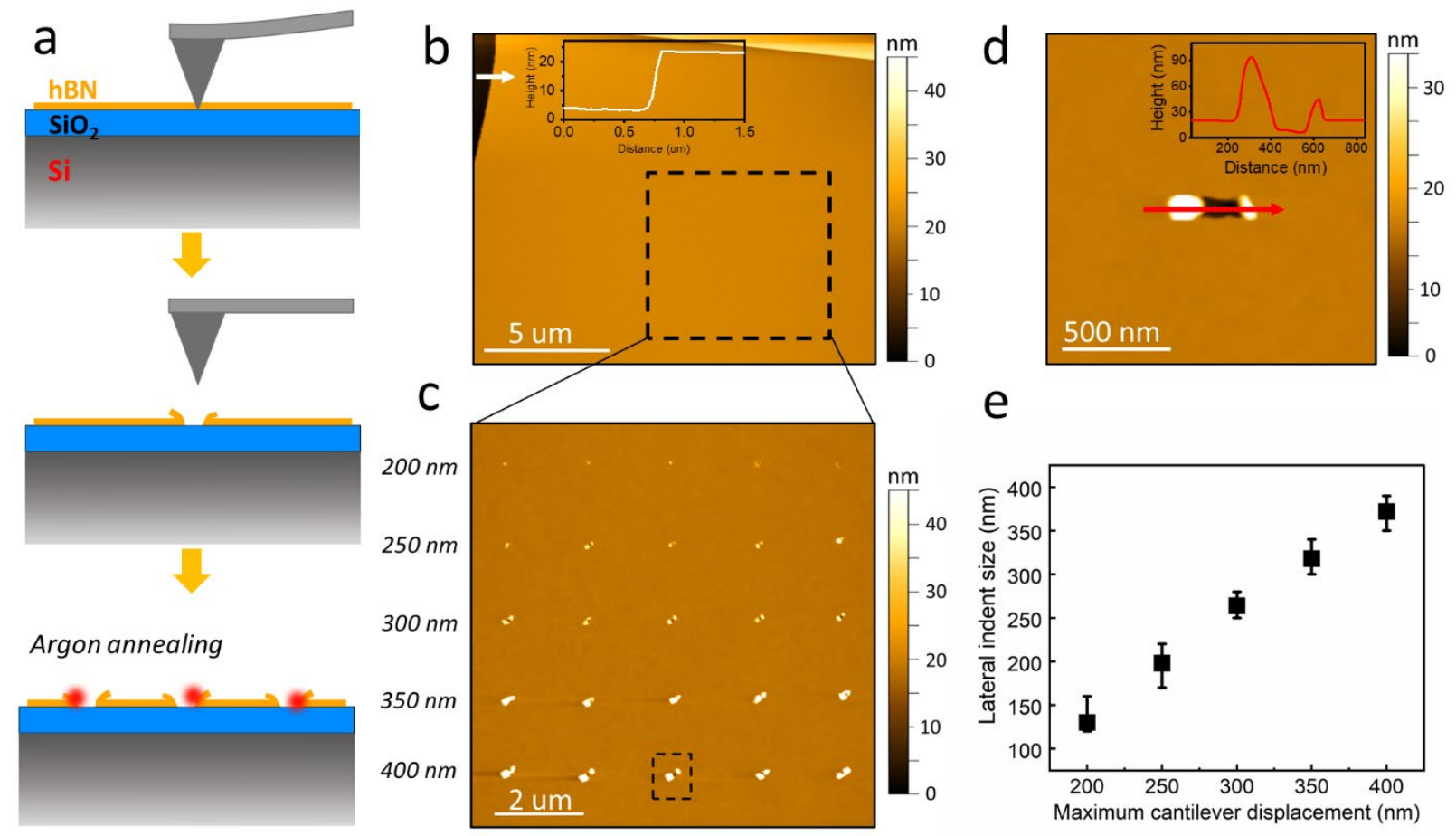

Figure 1. a) A schematic showing the activation of SPEs in $\mathrm{hBN}$ with the AFM nanoindentation technique followed by high temperature annealing in argon. b) An AFM image showing a thin hBN flake used for demonstrating the nanoindentation process. Upper inset: a height profile collected at the flake edge along the white arrow, showing a flake thickness of $19.8 \mathrm{~nm}$. c) an AFM image of the area inside the dashed box in $b)$ after nanoindentation. On the left lists the values of maximum cantilever displacement $\left(\Delta z_{\max }\right)$ during the indentation for each row of indents. d) An enlarged view of one specific indent inside the black dashed box in c), showing a dimple in the flake as well as two broken hBN areas next to the dimple. Note that the image was scanned at $45^{\circ}$ with respect to the image in c) to present the indent along the horizontal direction. Inset: a height profile collected across the indent along the red arrow. e) Dependence of the lateral indent size as a function of the maximum cantilever displacement $\Delta z_{\max }$.

Our findings are in contrast with reported AFM indentation experiments on TMDCs ${ }^{11,18}$, where TMDC layers are completely deformed into indented dips with depths depending on $\Delta \mathrm{z}_{\max }$, without being torn apart by cantilevers. One reason can be attributed to a relatively rigid substrate $\left(\mathrm{SiO}_{2}\right.$-on-Si) that is not amenable to deformation, while in ref ${ }^{11}$ a soft polymer substrate is used 
to facilitate the downward deformation of the TMDC layer. The difference between $\mathrm{hBN}$ and TMDCs in terms of their mechanical properties could also play an important role. High-quality hBN thin films around $15 \mathrm{~nm}$ thick are reported to have a Young's Modulus of 1.2 $\mathrm{TPa}^{19}$, which is more than 4 times larger than the Young's Modulus of TMDCs ${ }^{20}$. This indicates that $\mathrm{hBN}$ is a stiffer material than TMDCs and tends to break more easily before significant deformation. An additional factor is the different types of cantilevers used among studies in terms of coating material, tip radius, etc., which might lead to varied cantilever-sample interactions and sample responses. Here, the indentation of $\mathrm{hBN}$ flakes without notable damage into substrates is, in fact, of unique advantage in practical applications where hBN flakes are placed on photonic components such as waveguides, resonators, cavities, etc.

After the nanoindentation, an annealing step is essential to activate SPEs in hexagonal boron nitride and meanwhile remove possible organic residues from the exfoliation process. In this work, all samples are annealed in argon at $1000{ }^{\circ} \mathrm{C}$ for 30 minutes to achieve efficient SPE activation. $^{7}$ In the following experiments, two types of AFM indented hBN samples are annealed and characterized: one with varied indentation sizes on the same flake to investigate the correlation between indent sizes and SPE generation, and another with a single indent size combining a high SPE yield and indentation precision. Figure 2 a shows an $\mathrm{hBN}$ flake that has been indented with three different $\Delta z_{\max }$, namely, $250 \mathrm{~nm}$ (group 1), $500 \mathrm{~nm}$ (group 2) and $1500 \mathrm{~nm}$ (group 3). Here, relatively large step sizes in $\Delta \mathrm{z}_{\max }$ between groups are used compared to the demonstration in Figure 1, which helps us to extract the dependence of SPEs on the lateral indent size in a broader size range. The lateral size distribution of all indents can be found in Figure S3 (Supplementary Information). Average indent sizes of $200 \mathrm{~nm}, 400 \mathrm{~nm}$ and $750 \mathrm{~nm}$ are measured in the three groups above. It needs to be noted that an indent size of $750 \mathrm{~nm}$ for $\Delta z_{\max }=1500 \mathrm{~nm}$ no longer fits into the linear relationship shown in Figure 1e. However, fitting for indents larger than $500 \mathrm{~nm}$ is out of the scope of this work since smaller indents are preferred in practice. We also note that even for $\Delta \mathrm{z}_{\max }$ as large as $1500 \mathrm{~nm}$, the depth of resulting indents is still comparable to the $\mathrm{hBN}$ flake thickness (Figure S3d), again confirming that our technique has negligible impact on substrates. Overall, our indentation procedure can produce a designated indent size with decent repeatability. To further narrow down the size distribution of indents created by a given $\Delta z_{\max }$, one can explore the rich parameters in the nanoindentation procedure, such as the indentation trigger point, cantilever extension/retraction velocity, dwell time at $\Delta \mathrm{z}_{\max }$, etc. 

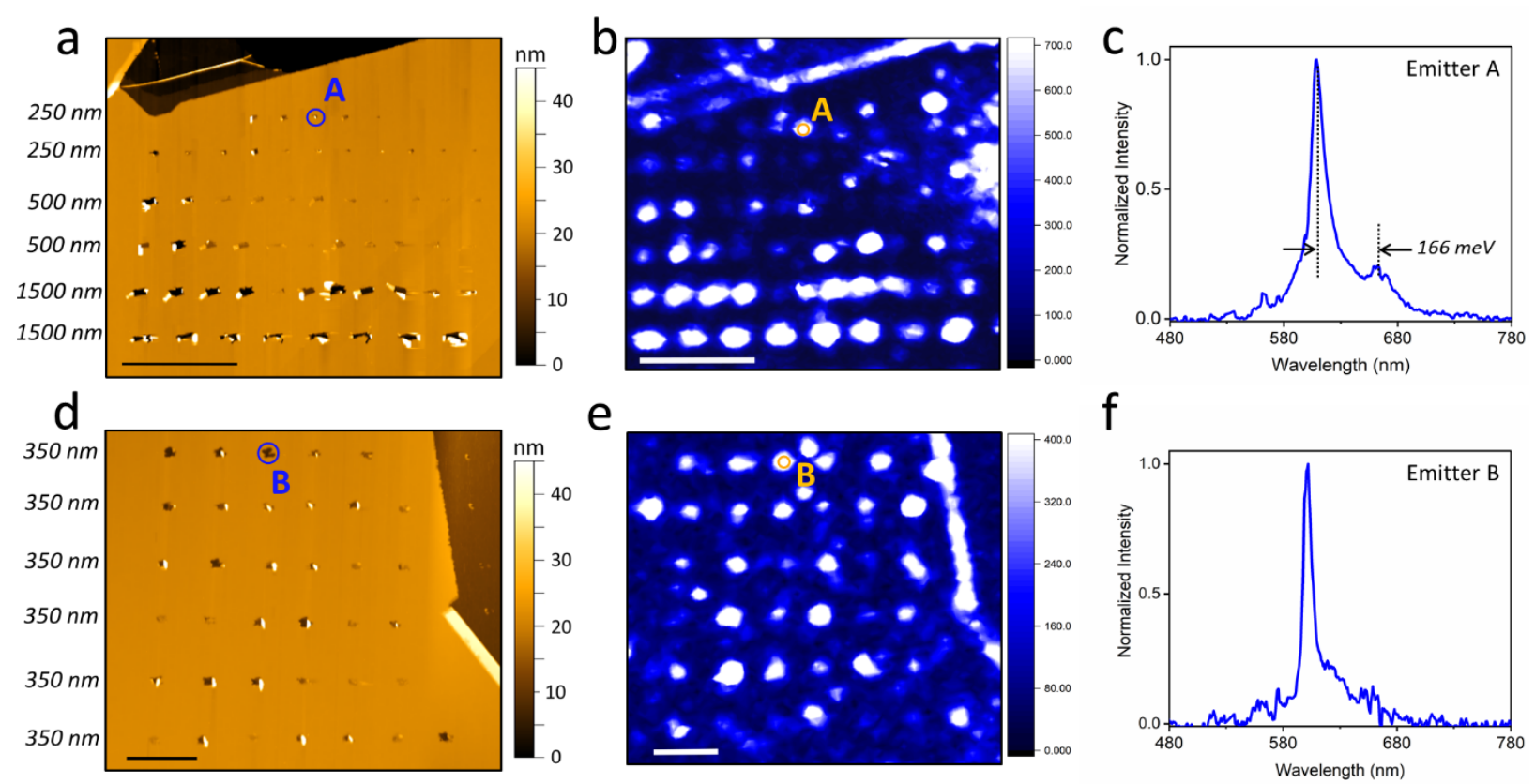

Figure 2. a) AFM image of the first flake after nanoindentation. Six rows of indents are created with the values of $\Delta z_{\max }$ indicated on the left of each row. From top to bottom, the number of indents in each row is 5, 11, 11, 9, 10 and 8, respectively. Scale bar: 5 um. b) Photoluminescence (PL) map from the same area shown in a) after Ar annealing. Scale bar: 5 um. c) Background-subtracted spectrum from a representative emitter A circled in a) and b). A phonon sideband (PSB) that is $166 \mathrm{meV}$ away from the zero-phonon line (ZPL) at $608 \mathrm{~nm}$ is identified. d) AFM image of the second flake after nanoindentation. Six rows of indents are created with the same $\Delta z_{\max }$ of $350 \mathrm{~nm}$. From top to bottom, the number of indents in each row is 5,6 , 6, 6, 6 and 7, respectively. Scale bar: 2 um. e) PL map of the same area shown in d) after Ar annealing. Scale bar: 2um. f) Background-subtracted spectrum from a representative emitter B circled in d) and e), showing a sharp ZPL line centered at $602 \mathrm{~nm}$.

After annealing in Ar, the flake is scanned with our home-built confocal microscope at room temperature. A 532-nm-wavelength continuous wave $(\mathrm{CW})$ pump laser is used for scanning at a laser power of $800 \mathrm{uW}$ and the photoluminescence (PL) map from the AFM-indented area is collected (Figure 2b). Bright, isolated spots are identified at positions corresponding to the indented areas, with low background emission rates away from these spots. Spectroscopic measurements reveal that PL spectra from those bright spots typically show well-defined emission peaks on top of a low background. The spectrum of a representative emitter A is presented in Figure 2c. There is a sharp emission peak at $608 \mathrm{~nm}(2.04 \mathrm{eV})$ that matches the one of the reported 
zero-phonon lines (ZPLs) of hBN emitters $(602 \mathrm{~nm}) .^{7} \mathrm{~A}$ broader peak that is $\sim 166 \mathrm{meV}$ away from the ZPL is recognized as a phonon sideband (PSB) corresponding to the in-plane optical phonons of $\mathrm{hBN}^{7,21-23}$. This confirms that our technique is capable to activate room-temperature emitters in $\mathrm{hBN}$ at indents as small as $200 \mathrm{~nm}$ in size.

Another important observation is that the yield of bright emission spots increases monotonically with the indent size (Figure $2 b$ ). While a high emitter yield would be desired, it is also preferable to work with emitters from relatively small indents such that the integration of emitters with photonic structures could be achieved with better precision ${ }^{24-26}$. To balance the above two factors, we nano-indented another flake with a maximum cantilever displacement of $350 \mathrm{~nm}$. Advantages of this specific $\Delta z_{\max }$ will be discussed later. The developed nanoindentation procedure has reproduced arrays of indents with comparable sizes (Figure 2d and Supplementary Information, Figure S4). An average indent size of $290 \mathrm{~nm}$ was measured along the lateral (horizontal) direction. PL map of this area (Figure 2e) shows arrays of bright emission spots that match well with the AFM indentation pattern. The PL spectrum of a typical emitter noted as emitter $B$ in the area (Figure 2f) has a sharp ZPL emission centered at $602 \mathrm{~nm}(2.06 \mathrm{eV})$.

After confirming the existence of hBN emitters at nano-indented sites on both flakes above, we next evaluated their single-photon purity using a Hanbury Brown and Twiss (HBT) setup that measures the second-order autocorrelation functions $\mathrm{g}^{2}(\mathrm{t})$ of emitter A and B (Figure 3a). Both emitters have $\mathrm{g}^{2}(0)<0.5$ at zero-delay time, confirming that they are single photon sources. The experimental $\mathrm{g}^{2}(\mathrm{t})$ data measured in our experiments can be well fitted with a three-level model expressed as: $: 5,27$

$$
g^{2}(t)=1-(1+a) \cdot e^{-\frac{|t|}{\tau_{1}}}+a \cdot e^{-\frac{|t|}{\tau_{2}}}
$$

where $\mathrm{a}$ is the photon bunching amplitude, while $\tau_{1}$ and $\tau_{2}$ are lifetimes of the excited and metastable states, respectively. After careful examination, 31 SPEs in total were identified out of all indented sites from the two hBN flakes above (Figure S5, Supplementary Information). The radiative decay lifetimes of all SPEs extracted from $\mathrm{g}^{2}(\mathrm{t})$ range from $1.17 \mathrm{~ns}$ to $7.88 \mathrm{~ns}$ with an average lifetime of $3.86 \mathrm{~ns}$ (Figure $3 \mathrm{~b}$ ). Our observation agrees with the typical lifetimes reported for room-temperature SPEs in hBN. ${ }^{7,28}$ To measure the brightness of these SPEs, PL intensities at a series of incident laser powers are recorded and then fitted using a first-order saturation model: $I=I_{\text {sat }} P /\left(P+P_{\text {sat }}\right)$, where $\mathrm{P}\left(\mathrm{P}_{\text {sat }}\right)$ and $\mathrm{I}\left(\mathrm{I}_{\text {sat }}\right)$ are the incident (saturated) power and $\mathrm{PL}$ intensity, respectively. Figure $3 \mathrm{c}$ shows the fitted saturation curve of one of the brightest emitters that 
saturates at $4.1 \mathrm{~mW}$ with a saturation intensity of $1.1 \mathrm{Mcps}$, comparable to the brightest $\mathrm{hBN}$ emitters created by other techniques. ${ }^{7,13,14,29}$ SPEs in this work typically exhibit linearly polarized emission (Figure 3c inset), indicating an atomic defect with in-plane dipole moment. ${ }^{28}$ The photostability of SPEs is examined by recording their PL time traces when excited by the $532 \mathrm{~nm}$ laser at $800 \mathrm{uW}$. It is observed that roughly $50 \%$ of the SPEs maintain stable emission during the optical characterization, while $40 \%$ and 10\% exhibit blinking and photobleaching behaviors, respectively (Figure S6, Supplementary Information). Blinking has been commonly observed in hBN SPEs ${ }^{12,21,30}$ as well as in other types of solid-state SPEs. ${ }^{31-33}$ It features switches between an "on" and "off" state with switching rates varying from one SPE to another. One important cause of blinking is the existence of other defects near the target SPE, which modifies the SPE charge state from time to time. According to previous reports, blinking is quite common for hBN SPEs created by deterministic techniques, ${ }^{8,12,13}$ even after high-temperature annealing which is proved to improve emission stability. ${ }^{29}$ This is likely related to the complex local environment near SPEs after the hBN lattice structures get modified deterministically by strain, electron/ion irradiation, etc. Strategies to effectively improve the photostability of deterministically formed hBN SPEs are therefore highly desirable. 

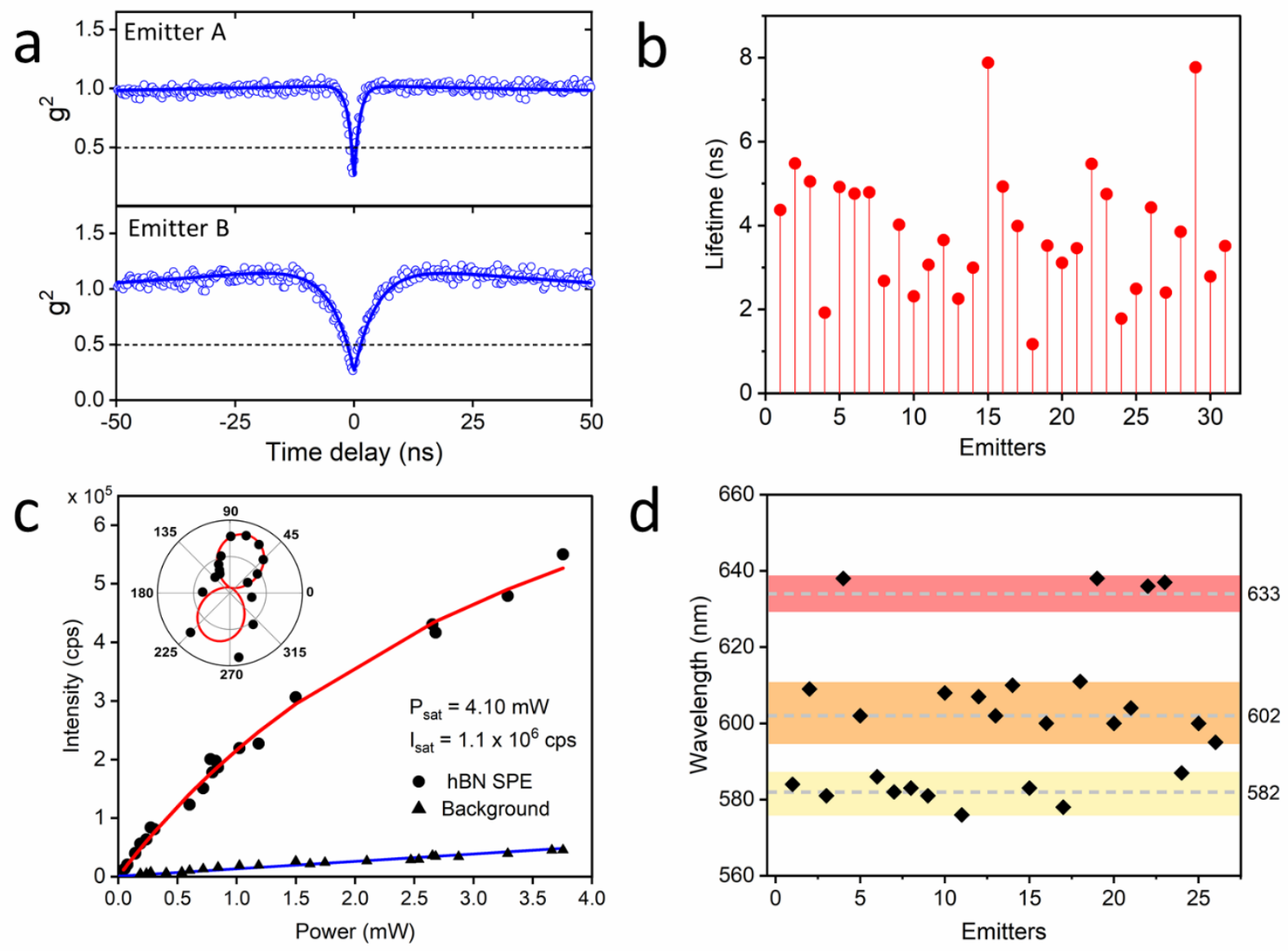

Figure 3. Photophysical properties of $\mathrm{hBN}$ emitters. a) Second-order autocorrelation measurements of emitter A \& B. Blue circles are experimental data while blue solid lines are fitted curves using the threelevel model. Both emitters show $\mathrm{g}^{2}(0)<0.5$ indicative of single photon emission. b) Distribution of radiative lifetimes from all $31 \mathrm{hBN}$ SPEs, extracted from $\mathrm{g}^{2}(\mathrm{t})$ fitting. The numbering of SPEs can be found in Figure S5 (Supplementary Information). c) Fluorescence saturation curve of a typical SPE with a saturation count of 1.1 Mcps. Inset: Emission polarization plot of a hBN SPE. d) Distribution of ZPLs for 26 photostable SPEs, revealing three classes of emitters with ZPLs at $582 \pm 6 \mathrm{~nm}, 602 \pm 9 \mathrm{~nm}$ and $633 \pm 5 \mathrm{~nm}$, respectively. The shaded bands are guides to the eye.

Figure $3 \mathrm{~d}$ summarizes the distribution of ZPLs for all photostable SPEs from AFMindented sites. The emitters can be classified into three groups with ZPLs centered at $582 \pm 6 \mathrm{~nm}$, $602 \pm 9 \mathrm{~nm}$ and $633 \pm 5 \mathrm{~nm}$, all of which match the previously reported ZPLs of hBN SPEs in the visible spectral range. ${ }^{7}$ One interesting fact is that, unlike SPEs created by other top-down techniques where a dominating portion of emitters show ZPLs at wavelengths shorter than 590 
$\mathrm{nm},{ }^{8,13}$ near $50 \%$ of emitters created with our technique exhibit ZPLs around $602 \mathrm{~nm}$ and another $40 \%$ show emission centered around $582 \mathrm{~nm} .35 \%$ of the SPEs show PSBs in their spectra that are $167 \pm 10 \mathrm{meV}$ away from their corresponding ZPLs (Figure S7, Supplementary Information), in agreement with previous reports. ${ }^{34-37}$ The relatively narrow distribution of ZPLs observed here implies that our technique is promising for deterministically creating hBN SPEs with predictable emission wavelengths.

Aside from SPEs, we also found emitters that possess some degrees of antibunching with $0.5<\mathrm{g}^{2}(0)<1$. Considering the low fluorescence background of our samples, such emitters are most likely composed of more than one SPEs within spatially unresolvable spots. ${ }^{38}$ Spectra collected from these emitters typically show broader emission peaks or multi-peaked emission, confirming the existence of multiple SPEs that could not be individually addressed (Figure S8, Supplementary Information). To demonstrate the correlation between $\mathrm{g}^{2}(0)$ and indent size of corresponding emitters, we summarized the distribution of $\mathrm{g}^{2}(0)$ values of emitters showing antibunched emission $\left(\mathrm{g}^{2}(0)<1\right)$ as a function of their lateral indent sizes. Emitters from both hBN flakes with average indent sizes of $200 \mathrm{~nm}, 290 \mathrm{~nm}, 400 \mathrm{~nm}$ and $740 \mathrm{~nm}$, are included for statistics. SPEs $\left(\mathrm{g}^{2}(0)<0.5\right)$ are found from all four groups of indents, while clustered emitters $\left(0.5<\mathrm{g}^{2}(0)\right.$ $<1$ ) are only found from indents with sizes $\geq 290 \mathrm{~nm}$ (Figure 4). After dividing the number of emitters with the total number of indents in each group, we obtained the emitter yields of both clustered emitters and SPEs for different indent sizes (bottom panel in Figure 4). The yield of clustered emitters shows a monotonic increase with indent sizes, from $0 \%$ for $200 \mathrm{~nm}$-sized indents to over $40 \%$ for an indent size of $740 \mathrm{~nm}$. In contrast, the SPE yield first increases with indent sizes, from $25 \%$ for indents of $200 \mathrm{~nm}$ up to $36 \%$ for indents of $400 \mathrm{~nm}$, then drops for indents larger than $400 \mathrm{~nm}$. The SPE yields for indents of $290 \mathrm{~nm}(32 \%)$ and $400 \mathrm{~nm}(36 \%)$ are, to the best of our knowledge, among the highest values ever reported for hBN SPEs created by top-down methods including FIB and nanopillar arrays. ${ }^{8,13}$ Specifically, indents around $290 \mathrm{~nm}$ combine a high SPE yield and relatively small feature size, making them promising for the deterministic integration of hBN SPEs with photonic/plasmonic structures; The effective generation of clustered emitters in larger indents $(740 \mathrm{~nm})$ could be useful as well, for example, for sensing applications. 


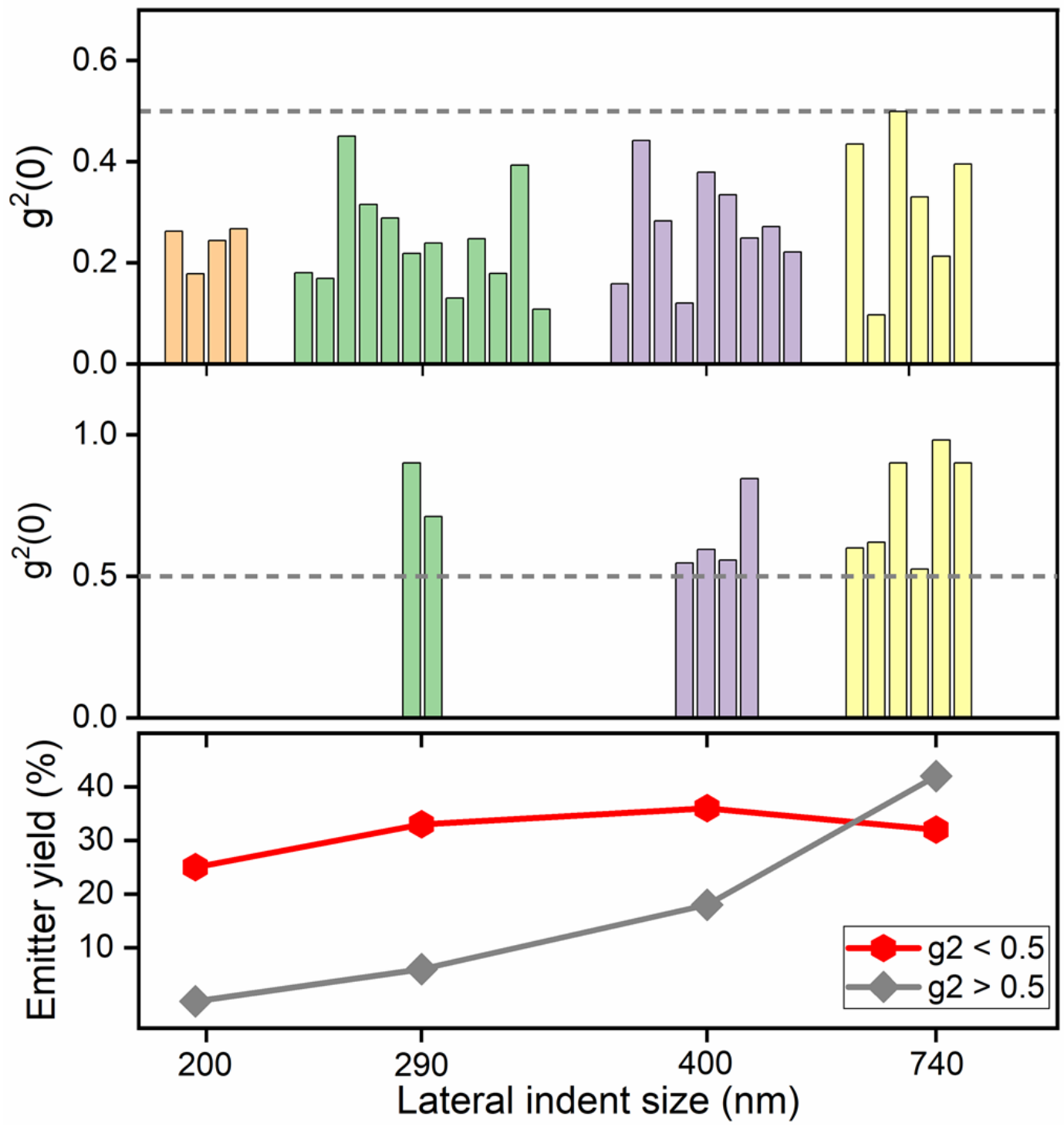

Figure 4. Top and middle panel: $\mathrm{g}^{2}(0)$ values of SPEs $\left(\mathrm{g}^{2}(0)<0.5\right.$, top) and clustered SPEs $\left(0.5<\mathrm{g}^{2}(0)<\right.$ 1, middle) as a function of corresponding indent sizes. Bottom panel: Yield of SPE and clustered emitters as a function of lateral indent size, calculated by dividing the number of emitters over the total number of indents created for each indent size.

We now discuss the formation mechanisms of $\mathrm{hBN}$ emitters created by AFM nanoindentation and their potential applications. First of all, the necessity of high-temperature annealing for SPE activation indicates that the emitters are most likely vacancy-related atomic defects as vacancy diffusion typically dominates at high temperatures. ${ }^{39}$ To validate this, we performed a control experiment by annealing additional nano-indented hBN samples in argon at a 
lower temperature, namely, $800^{\circ} \mathrm{C}$ (see Supplementary Information for details). As seen in Figure S9, SPEs are found near the indents but with much lower emitter yields compared to hBN flakes annealed at $1000^{\circ} \mathrm{C}$. This confirms that the formation of SPEs is less favorable at lower annealing temperatures likely due to the reduced vacancy diffusion rate. Our observation agrees with $\mathrm{ref}^{7}$ where a monotonic increase in emitter yield is reported as a function of annealing temperature. However, further increasing the annealing temperature above $1000^{\circ} \mathrm{C}$ is not preferred in practice considering the degradation of involved materials. Other post-AFM treatments such as plasma etching could be explored as alternative ways to achieve higher SPE yields. As been reported previously, plasma treatments is capable of generating vacancies in $\mathrm{hBN}$ lattices as well as creating hBN SPEs. ${ }^{29,40,41}$ Adding a plasma etching step after AFM nanoindentation might help to improve the SPE yield by increasing the probability of forming vacancy-related defects that act as SPEs near the indents.

The determination of hBN SPE locations with nanoscale precision has not be achieved here due to the diffraction-limited resolution of the confocal microscope. Nevertheless, we infer that SPEs are most likely formed either at the edge of indented dips or on the bent, delaminated $\mathrm{hBN}$ areas next to the dips. The former favors SPE formation as defects tend to accumulate at structure/grain boundaries, ${ }^{13,42}$ while the latter could activate strain-induced SPEs due to the large curvature near the bent $\mathrm{hBN}$ areas. It is highly possible that both mechanisms play a role here, resulting in higher SPE yields than those reported for the at-the-boundary creation or strain engineering. ${ }^{8,13}$ To locate SPEs with a higher resolution, one can use a near-field scanning optical microscope (NSOM) ${ }^{43}$ scanning antenna microscope ${ }^{44}$ or combined confocal microscope-AFM setup $^{45}$ to get correlated PL and topographical maps.

One of the most important advantages of hBN SPEs activated with AFM indentation is the combined high SPE yield and high-precision position control. As been demonstrated above, a SPE yield of $32 \%$ is obtained for indents less than $300 \mathrm{~nm}$ in dimension, which sets the new record for deterministic creation of hBN SPEs on non-structured substrates. It also needs to be emphasized that indents created by AFM features sharp, well-defined edges, in contrast to holes/spots induced by radiation methods that typically have poorly defined boundaries due to the diffuse nature of radiation beams. On the other hand, our technique can be extended to hBN flakes with various thicknesses by simply adjusting the indentation parameters, making it more versatile than strain 
engineering with structured substrates as the latter typically requires thin hBN films to achieve desired deformation.

The position-controlled creation of hBN SPEs with AFM nanoindentation could be a promising route for various quantum photonic applications. For example, it enables the efficient on-chip integration of hBN SPEs with photonic waveguides or cavities by creating SPEs at desired positions, in contrast to previously studies where hBN SPEs are coupled to photonic structures either randomly ${ }^{25,46-48}$ or more deterministically, yet with extensive alignment efforts. ${ }^{49}$ The developed technique also offers a great potential for coupling hBN SPEs to plasmonic nanostructures to achieve strong emission enhancement. ${ }^{50}$ Compared to randomly activated SPEs in hBN that do not possess recognizable topographical features, SPEs near the nano-sized indents make it possible to deterministically assemble plasmonic cavity/antenna structures ${ }^{51,52}$ with high SPE coupling efficiency. In addition, AFM-induced hBN SPEs could be used in other studies on hBN SPEs that might benefit from deterministic emitter coupling, such as sensing, emission tuning, ${ }^{53,54}$ quantum non-linear optics, ${ }^{55,56}$ and more.

\section{Conclusion}

We demonstrated a new route to deterministically create room-temperature SPEs in $\mathrm{hBN}$ utilizing AFM nanoindentation. The technique is applied to thin hBN flakes on an unpatterned $\mathrm{SiO}_{2}$-coated $\mathrm{Si}$ substrate. By carefully controlling the indentation parameters, indents with lateral sizes ranging from $200 \mathrm{~nm}$ to over $700 \mathrm{~nm}$ are obtained, without notable damage or deformation of the substrate. After high temperature annealing in argon, hBN SPEs are activated near the indents for various indent sizes. The SPEs show a relatively narrow distribution of emission wavelengths, with over $80 \%$ of them emitting around $583 \mathrm{~nm}$ and $602 \mathrm{~nm}$. A maximum SPE yield of $36 \%$ is obtained for an indent size of $400 \mathrm{~nm}$, and smaller indents around $290 \mathrm{~nm}$ give a comparably high SPE yield. Our method involves no lithographic or other patterning steps, so that fab-induced fluorescence contamination is avoided. While the nature of these indentation induced SPEs has to be studied in more detail, it is inferred that the creation of structural edges and highly strained hBN areas near the indents could be responsible for the observed high SPE yield. The efficient SPE activation on flat, chip-compatible substrates with high precision allows controlled coupling of hBN SPEs with plasmonic and photonic devices for various quantum information 
applications. Hence, our results open exciting avenues towards the on-chip deterministic integration of hBN SPEs and future on-chip quantum photonic devices.

\section{Materials and Methods}

\section{Sample Preparation}

hBN flakes were obtained using a standard mechanical exfoliation method. High-quality hBN crystals produced by high-pressure synthesis were picked up by a sticky tape and then exfoliated to new tapes multiple times to get thin flakes. After several exfoliations, thin hBN flakes were transferred to flat silicon substrates coated with a $285 \mathrm{~nm}$-thick $\mathrm{SiO}_{2}$ layer. The substrates were cleaned with acetone, methanol, isopropanol and de-ionized water both before and after the transfer procedure. After transferring, an optical microscope was used to identify and locate hBN thin flakes based on the thickness-dependent reflection colors of hBN. The exact thicknesses of those thin flakes were then measured by an atomic force microscope (Cypher S AFM, Asylum Research). Only smooth flakes (without folds or wrinkles) with thicknesses less than $25 \mathrm{~nm}$ were selected for the rest of experiments.

Nanoindentation on pre-selected hBN flakes was carried out with the same AFM operated in the contact mode. Cantilevers with a diamond-like carbon coating layer (Tap300DLC, $300 \mathrm{kHz}$, $40 \mathrm{~N} / \mathrm{m}$, BudgetSensors) were used. The indentation was triggered after the cantilever got in contact with the sample surface and the deflection (measured on the photo-detector, in the unite of voltage) reached $0.3 \mathrm{~V}$. The maximum cantilever displacement along the vertical direction was adjustable from $0 \mathrm{~nm}$ up to $1500 \mathrm{~nm}$ to create indents with different lateral sizes. A displacement velocity of $100 \mathrm{~nm} / \mathrm{s}$ was used for both the extension and retraction of cantilevers, with a dwell time of $2 \mathrm{~s}$ at the maximum displacement position. After arrays of indents were generated, the AFM was switched to tapping mode to get topographical images of the indented areas.

Argon annealing was performed in a tube furnace (Lindberg/Blue M) following the indentation experiments. The samples were heated from room temperature to $1000^{\circ} \mathrm{C}$ with a ramping time of $\sim 40$ mins. After being held at $1000^{\circ} \mathrm{C}$ for $30 \mathrm{mins}$ in a continuous argon gas flow, the samples were allowed slowly cool down to room temperature in 6 hours.

\section{Optical Characterization}


Optical characterization was performed by a home-built scanning confocal microscopy setup based on a commercially available inverted microscope body (Nikon Ti-U) with the objective scanned by a P-561 PIMars piezo stage with an E-712 controller and Alignment Firmware (Physik Instrumente). Optical pumping for continuous wave (CW) excitation experiments was provided by a $200 \mathrm{~mW}$ continuous wave $532 \mathrm{~nm}$ laser (RGB Photonics). The fluorescence to be detected was separated from the pump radiation by a main dichroic beamsplitter (550 nm long-pass DMLP550L, Thorlabs), and subsequently a $550 \mathrm{~nm}$ long-pass filter (FEL0550, Thorlabs). The collected fluorescence was passed through a 100 um pinhole. Two single-photon avalanche detectors (SPADs) with a $30 \mathrm{ps}$ time resolution and 35\% quantum efficiency at $650 \mathrm{~nm}$ (PDM, Micro-Photon Devices) were used for autocorrelation measurements. An SPAD with 69\% quantum efficiency at $650 \mathrm{~nm}$ (SPCM-AQRH, Excelitas) was used for scanning and saturation measurements. Spectral measurements were performed with a QE65000 visible-to-near infrared spectrometer (Ocean Insight). To measure the emission polarization, a broadband analyzer (LPVISC050-MP2, Thorlabs) was placed in the optical collection path and rotated in $20^{\circ}$ increments. A time trace of the emission intensity was recorded, and averaged photon counts were correlated with analyzer angles.

\section{Acknowledgements}

The authors acknowledge S. I. Bogdanov, A. Senichev and A. B. Solanki for helpful discussions on the optical characterization of hBN SPEs. This work is supported by the U.S. Department of Energy (DOE), Office of Science through the Quantum Science Center (QSC), a National Quantum Information Science Research Center and National Science Foundation Award 2015025ECCS.

\section{References}

(1) O’Brien, J. L.; Furusawa, A.; Vučković, J. Photonic Quantum Technologies. Nature Photonics. 2009, 3, 687-695.

(2) Aharonovich, I.; Englund, D.; Toth, M. Solid-State Single-Photon Emitters. Nature Photonics. 2016. 10, 631-641. 
(3) Atatüre, M.; Englund, D.; Vamivakas, N.; Lee, S. Y.; Wrachtrup, J. Material Platforms for Spin-Based Photonic Quantum Technologies. Nature Reviews Materials. 2018, 3, 38-51.

(4) He, Y. M.; Clark, G.; Schaibley, J. R.; He, Y.; Chen, M. C.; Wei, Y. J.; Ding, X.; Zhang, Q.; Yao, W.; Xu, X.; Lu, C. Y.; Pan, J. W. Single Quantum Emitters in Monolayer Semiconductors. Nat. Nanotechnol. 2015, 10, 497-502.

(5) Tran, T. T.; Bray, K.; Ford, M. J.; Toth, M.; Aharonovich, I. Quantum Emission from Hexagonal Boron Nitride Monolayers. Nat. Nanotechnol. 2016, 11, 37-41.

(6) Bourrellier, R.; Meuret, S.; Tararan, A.; Stéphan, O.; Kociak, M.; Tizei, L. H. G.; Zobelli, A. Bright UV Single Photon Emission at Point Defects in H-BN. Nano Lett. 2016, 16, 4317-4321.

(7) Tran, T. T.; Elbadawi, C.; Totonjian, D.; Lobo, C. J.; Grosso, G.; Moon, H.; Englund, D. R.; Ford, M. J.; Aharonovich, I.; Toth, M. Robust Multicolor Single Photon Emission from Point Defects in Hexagonal Boron Nitride. ACS Nano 2016, 10, 7331-7338.

(8) Proscia, N. V.; Shotan, Z.; Jayakumar, H.; Reddy, P.; Dollar, M.; Alkauskas, A.; Doherty, M.; Meriles, C. A.; Menon, V. M. Near-Deterministic Activation of Room Temperature Quantum Emitters in Hexagonal Boron Nitride. Optica. 2018, 5, 1128-1134.

(9) Li, H.; Contryman, A. W.; Qian, X.; Ardakani, S. M.; Gong, Y.; Wang, X.; Weisse, J. M.; Lee, C. H.; Zhao, J.; Ajayan, P. M.; Li, J.; Manoharan, H. C.; Zheng, X. Optoelectronic Crystal of Artificial Atoms in Strain-Textured Molybdenum Disulphide. Nat. Commun. 2015, 6, 7381 .

(10) Tonndorf, P.; Schmidt, R.; Schneider, R.; Kern, J.; Buscema, M.; Steele, G. A.; Castellanos-Gomez, A.; van der Zant, H. S. J.; Michaelis de Vasconcellos, S.; Bratschitsch, R. Single-Photon Emission from Localized Excitons in an Atomically Thin Semiconductor. Optica 2015, 2, 347-352.

(11) Rosenberger, M. R.; Dass, C. K.; Chuang, H. J.; Sivaram, S. V.; McCreary, K. M.; Hendrickson, J. R.; Jonker, B. T. Quantum Calligraphy: Writing Single-Photon Emitters in a Two-Dimensional Materials Platform. ACS Nano 2019, 13, 904-912. Li, C.; Mendelson, N.; Ritika, R.; Chen, Y.-L.; Xu, Z.-Q.; Toth, M.; Aharonovich, I. 
Scalable and Deterministic Fabrication of Quantum Emitter Arrays from Hexagonal Boron Nitride. Nano Lett. 2021, 21, 3626-3632.

(13) Ziegler, J.; Klaiss, R.; Blaikie, A.; Miller, D.; Horowitz, V. R.; Alemán, B. J.

Deterministic Quantum Emitter Formation in Hexagonal Boron Nitride via Controlled Edge Creation. Nano Lett. 2019, 19, 2121-2127.

(14) Fournier, C.; Plaud, A.; Roux, S.; Pierret, A.; Rosticher, M.; Watanabe, K.; Taniguchi, T.; Buil, S.; Quélin, X.; Barjon, J.; Hermier, J. P.; Delteil, A. Position-Controlled Quantum Emitters with Reproducible Emission Wavelength in Hexagonal Boron Nitride. Nat. Commun. 2021, 12, 3779.

(15) Shahmoon, E.; Wild, D. S.; Lukin, M. D.; Yelin, S. F. Cooperative Resonances in Light Scattering from Two-Dimensional Atomic Arrays. Phys. Rev. Lett. 2017, 118, 113601.

(16) Rui, J.; Wei, D.; Rubio-Abadal, A.; Hollerith, S.; Zeiher, J.; Stamper-Kurn, D. M.; Gross, C.; Bloch, I. A Subradiant Optical Mirror Formed by a Single Structured Atomic Layer. Nature 2020, 583, 369-374.

(17) Bettles, R. J.; Gardiner, S. A.; Adams, C. S. Enhanced Optical Cross Section via Collective Coupling of Atomic Dipoles in a 2D Array. Phys. Rev. Lett. 2016, 116, 103602.

(18) Kumar, P.; Balakrishnan, V. Nanosculpting of Atomically Thin 2D Materials for SiteSpecific Photoluminescence Modulation. Adv. Opt. Mater. 2018, 6, 1701284.

(19) Kim, S. M.; Hsu, A.; Park, M. H.; Chae, S. H.; Yun, S. J.; Lee, J. S.; Cho, D. H.; Fang, W.; Lee, C.; Palacios, T.; Dresselhaus, M.; Kim, K. K.; Lee, Y. H.; Kong, J. Synthesis of Large-Area Multilayer Hexagonal Boron Nitride for High Material Performance. Nat. Commun. 2015, 6, 8662 .

(20) Falin, A.; Holwill, M.; Lv, H.; Gan, W.; Cheng, J.; Zhang, R.; Qian, D.; Barnett, M. R.; Santos, E. J. G.; Novoselov, K. S.; Tao, T.; Wu, X.; Li, L. H. Mechanical Properties of Atomically Thin Tungsten Dichalcogenides: $\mathrm{WS}_{2}, \mathrm{WSe}_{2}$, and $\mathrm{WTe}_{2}$. ACS Nano 2021, 15 , 2600-2610.

(21) Shotan, Z.; Jayakumar, H.; Considine, C. R.; Mackoit, M.; Fedder, H.; Wrachtrup, J.; Alkauskas, A.; Doherty, M. W.; Menon, V. M.; Meriles, C. A. Photoinduced Modification 
of Single-Photon Emitters in Hexagonal Boron Nitride. ACS Photonics 2016, 3, 2490 2496.

(22) Jung, S.; Park, M.; Park, J.; Jeong, T. Y.; Kim, H. J.; Watanabe, K.; Taniguchi, T.; Ha, D. H.; Hwang, C.; Kim, Y. S. Vibrational Properties of H-BN and h-BN-Graphene Heterostructures Probed by Inelastic Electron Tunneling Spectroscopy. Sci. Rep. 2015, 5, 16642.

(23) Wang, Q.; Zhang, Q.; Zhao, X.; Luo, X.; Wong, C. P. Y.; Wang, J.; Wan, D.; Venkatesan, T.; Pennycook, S. J.; Loh, K. P.; Eda, G.; Wee, A. T. S. Photoluminescence Upconversion by Defects in Hexagonal Boron Nitride. Nano Lett. 2018, 18, 6898-6905.

(24) Caldwell, J. D.; Aharonovich, I.; Cassabois, G.; Edgar, J. H.; Gil, B.; Basov, D. N. Photonics with Hexagonal Boron Nitride. Nature Reviews Materials. 2019, 4, 552-567.

(25) Kim, S.; Duong, N. M. H.; Nguyen, M.; Lu, T. J.; Kianinia, M.; Mendelson, N.; Solntsev, A.; Bradac, C.; Englund, D. R.; Aharonovich, I. Integrated on Chip Platform with Quantum Emitters in Layered Materials. Adv. Opt. Mater. 2019, 7, 1901132.

(26) Proscia, N. V.; Jayakumar, H.; Ge, X.; Lopez-Morales, G.; Shotan, Z.; Zhou, W.; Meriles, C. A.; Menon, V. M. Microcavity-Coupled Emitters in Hexagonal Boron Nitride. Nanophotonics 2020, 9, 2937-2944.

(27) Martínez, L. J.; Pelini, T.; Waselowski, V.; Maze, J. R.; Gil, B.; Cassabois, G.; Jacques, V. Efficient Single Photon Emission from a High-Purity Hexagonal Boron Nitride Crystal. Phys. Rev. B 2016, 94, 121405(R).

(28) Exarhos, A. L.; Hopper, D. A.; Grote, R. R.; Alkauskas, A.; Bassett, L. C. Optical Signatures of Quantum Emitters in Suspended Hexagonal Boron Nitride. ACS Nano 2017, $11,3328-3336$.

(29) Xu, Z. Q.; Elbadawi, C.; Tran, T. T.; Kianinia, M.; Li, X.; Liu, D.; Hoffman, T. B.; Nguyen, M.; Kim, S.; Edgar, J. H.; Wu, X.; Song, L.; Ali, S.; Ford, M.; Toth, M.; Aharonovich, I. Single Photon Emission from Plasma Treated 2D Hexagonal Boron Nitride. Nanoscale 2018, 10, 7957-7965. Li, X.; Shepard, G. D.; Cupo, A.; Camporeale, N.; Shayan, K.; Luo, Y.; Meunier, V.; 
Strauf, S. Nonmagnetic Quantum Emitters in Boron Nitride with Ultranarrow and Sideband-Free Emission Spectra. ACS Nano 2017, 11, 6652-6660.

(31) Tang, J.; Marcus, R. A. Mechanisms of Fluorescence Blinking in Semiconductor Nanocrystal Quantum Dots. J. Chem. Phys. 2005, 123, 054704.

(32) Ai, N.; Walden-Newman, W.; Song, Q.; Kalliakos, S.; Strauf, S. Suppression of Blinking and Enhanced Exciton Emission from Individual Carbon Nanotubes. ACS Nano 2011, 5, 2664-2670.

(33) Bradac, C.; Gaebel, T.; Naidoo, N.; Sellars, M. J.; Twamley, J.; Brown, L. J.; Barnard, A. S.; Plakhotnik, T.; Zvyagin, A. V.; Rabeau, J. R. Observation and Control of Blinking Nitrogen-Vacancy Centres in Discrete Nanodiamonds. Nat. Nanotechnol. 2010, 5, 345349.

(34) Boll, M. K.; Radko, I. P.; Huck, A.; Andersen, U. L. Photophysics of Quantum Emitters in Hexagonal Boron-Nitride Nano-Flakes. Opt. Express 2020, 28, 7475.

(35) Feldman, M. A.; Puretzky, A.; Lindsay, L.; Tucker, E.; Briggs, D. P.; Evans, P. G.; Haglund, R. F.; Lawrie, B. J. Phonon-Induced Multicolor Correlations in HBN SinglePhoton Emitters. Phys. Rev. B 2019, 99, 020101(R).

(36) Kianinia, M.; Regan, B.; Tawfik, S. A.; Tran, T. T.; Ford, M. J.; Aharonovich, I.; Toth, M. Robust Solid-State Quantum System Operating at 800 K. ACS Photonics 2017, 4, 768773.

(37) Jungwirth, N. R.; Calderon, B.; Ji, Y.; Spencer, M. G.; Flatté, M. E.; Fuchs, G. D. Temperature Dependence of Wavelength Selectable Zero-Phonon Emission from Single Defects in Hexagonal Boron Nitride. Nano Lett. 2016, 16, 6052-6057.

(38) Brouri, R.; Beveratos, A.; Poizat, J.-P.; Grangier, P. Photon Antibunching in the Fluorescence of Individual Color Centers in Diamond. Opt. Lett. 2000, 25, 1294.

(39) Pezzagna, S.; Rogalla, D.; Wildanger, D.; Meijer, J.; Zaitsev, A. Creation and Nature of Optical Centres in Diamond for Single-Photon Emission-Overview and Critical Remarks. New J. Phys. 2011, 13, 035024. 
(40) Singh, R. S.; Tay, R. Y.; Chow, W. L.; Tsang, S. H.; Mallick, G.; Teo, E. H. T. Band Gap Effects of Hexagonal Boron Nitride Using Oxygen Plasma. Appl. Phys. Lett. 2014, 104, 163101.

(41) Petravic, M.; Peter, R.; Kavre, I.; Li, L. H.; Chen, Y.; Fan, L. J.; Yang, Y. W. Decoration of Nitrogen Vacancies by Oxygen Atoms in Boron Nitride Nanotubes. Phys. Chem. Chem. Phys. 2010, 12, 15349-15353.

(42) Choi, S.; Tran, T. T.; Elbadawi, C.; Lobo, C.; Wang, X.; Juodkazis, S.; Seniutinas, G.; Toth, M.; Aharonovich, I. Engineering and Localization of Quantum Emitters in Large Hexagonal Boron Nitride Layers. ACS Appl. Mater. Interfaces 2016, 8, 29642-29648.

(43) Dürig, U.; Pohl, D. W.; Rohner, F. Near-Field Optical-Scanning Microscopy. J. Appl. Phys. 1986, 59, 3318-3327.

(44) Palombo Blascetta, N.; Liebel, M.; Lu, X.; Taniguchi, T.; Watanabe, K.; Efetov, D. K.; Van Hulst, N. F. Nanoscale Imaging and Control of Hexagonal Boron Nitride Single Photon Emitters by a Resonant Nanoantenna. Nano Lett. 2020, 20, 1992-1999.

(45) Gutiérrez-Arzaluz, L.; Ahmed, G. H.; Yang, H.; Shikin, S.; Bakr, O. M.; Malko, A. V.; Mohammed, O. F. Correlation of Photoluminescence and Structural Morphologies at the Individual Nanoparticle Level. J. Phys. Chem. A 2020, 124, 4855-4860.

(46) Fröch, J. E.; Kim, S.; Mendelson, N.; Kianinia, M.; Toth, M.; Toth, M.; Aharonovich, I.; Aharonovich, I. Coupling Hexagonal Boron Nitride Quantum Emitters to Photonic Crystal Cavities. ACS Nano 2020, 14, 7085-7091.

(47) Kim, S.; Fröch, J. E.; Christian, J.; Straw, M.; Bishop, J.; Totonjian, D.; Watanabe, K.; Taniguchi, T.; Toth, M.; Aharonovich, I. Photonic Crystal Cavities from Hexagonal Boron Nitride. Nat. Commun. 2018, 9, 2623.

(48) Schell, A. W.; Takashima, H.; Tran, T. T.; Aharonovich, I.; Takeuchi, S. Coupling Quantum Emitters in 2D Materials with Tapered Fibers. ACS Photonics 2017, 4, 761-767.

(49) Elshaari, W. A.; Skalli, A.; Gyger, S.; Nurizzo, M.; Schweickert, L.; Zadeh, E. I.; Svedendahl, M.; Steinhauer, S.; Zwiller, V. Deterministic Integration of HBN Emitter in Silicon NitridePhotonic Waveguide. Adv. Quantum Rechn. 2021, 2100032. 
(50) Tran, T. T.; Wang, D.; Xu, Z. Q.; Yang, A.; Toth, M.; Odom, T. W.; Aharonovich, I. Deterministic Coupling of Quantum Emitters in 2D Materials to Plasmonic Nanocavity Arrays. Nano Lett. 2017, 17, 2634-2639.

(51) Bogdanov, S. I.; Shalaginov, M. Y.; Lagutchev, A. S.; Chiang, C. C.; Shah, D.; Baburin, A. S.; Ryzhikov, I. A.; Rodionov, I. A.; Kildishev, A. V.; Boltasseva, A.; Shalaev, V. M. Ultrabright Room-Temperature Sub-Nanosecond Emission from Single Nitrogen-Vacancy Centers Coupled to Nanopatch Antennas. Nano Lett. 2018, 18, 4837-4844.

(52) Bogdanov, S. I.; Makarova, O. A.; Xu, X.; Martin, Z. O.; Lagutchev, A. S.; Olinde, M.; Shah, D.; Chowdhury, S. N.; Gabidullin, A. R.; Ryzhikov, I. A.; Rodionov, I. A.; Kildishev, A. V.; Bozhevolnyi, S. I.; Boltasseva, A.; Shalaev, V. M.; Khurgin, J. B. Ultrafast Quantum Photonics Enabled by Coupling Plasmonic Nanocavities to Strongly Radiative Antennas. Optica 2020, 7, 463-469.

(53) Mendelson, N.; Xu, Z. Q.; Tran, T. T.; Kianinia, M.; Scott, J.; Bradac, C.; Aharonovich, I.; Toth, M. Engineering and Tuning of Quantum Emitters in Few-Layer Hexagonal Boron Nitride. ACS Nano 2019, 13, 3132-3140.

(54) Mendelson, N.; Doherty, M.; Toth, M.; Aharonovich, I.; Tran, T. T. Strain-Induced Modification of the Optical Characteristics of Quantum Emitters in Hexagonal Boron Nitride. Adv. Mater. 2020, 32, 1908316.

(55) Schell, A. W.; Tran, T. T.; Takashima, H.; Takeuchi, S.; Aharonovich, I. Non-Linear Excitation of Quantum Emitters in Hexagonal Boron Nitride Multiplayers. APL Photonics 2016, $1,091302$.

(56) Chen, B.; He, Z.; Liu, Z. J.; Wang, Y. K.; Gao, Y. N.; Aharonovich, I.; Xu, Z. Q.; Liu, J. Simultaneously Enhanced Linear and Nonlinear Photon Generations from $\mathrm{WS}_{2}$ by Using Dielectric Circular Bragg Resonators. Nanophotonics 2020, 9, 2587-2592. 


\section{Supplementary Information}

\section{Creating Quantum Emitters in Hexagonal Boron Nitride Deterministically on Chip-Compatible Substrates}

Xiaohui Xul ${ }^{1}$ ZZachariah O. Martin ${ }^{2}$, Demid Sychev ${ }^{2}$, Alexei S. Lagutchev ${ }^{2}$, Yong Chen 2,3,4,

Takashi Taniguch ${ }^{5}$, Kenji Watanabe $^{5}$, Vladimir M. Shalaev ${ }^{2}$, Alexandra Boltasseval,2

${ }^{1}$ School of Materials Engineering, Purdue University, USA

${ }^{2}$ School of Electrical and Computer Engineering, Purdue University, USA

${ }^{3}$ Department of Physics and Astronomy, Purdue University, USA

${ }^{4}$ Department of Physics and Astronomy, Aarhus University, Denmark

${ }^{5}$ National Institute for Materials Science, Japan

\section{Contents}

I. Measurement and determination of lateral indent size

II. Dependence of the indentation depth on the maximum cantilever displacement ......... 23

III. Additional data from hBN flakes used for optical measurement .................................. 25

IV. Control experiment with hBN flakes annealed at $800^{\circ} \mathrm{C}$................................................... 29

References................................................................................................................................................. 31 


\section{Measurement and determination of lateral indent size}

Here we specify the method used to measure the lateral size of AFM-induced indents in this work. It is known that the AFM probes create various types of artifacts in AFM scanning/imaging. ${ }^{1}$ Specifically, when scanning a feature that is lower than the surface, such as an indent as in this work, the lateral size of the indent can appear smaller than its actual size due to the width of the AFM probe. ${ }^{2}$ The effect is illustrated in Figure S1a. Therefore, rather than taking the length of the recessed portion of the AFM height profile as the indent size, we denote the lateral indent size to be the distance between the two highest features across the indent, as shown in Figure S1b.
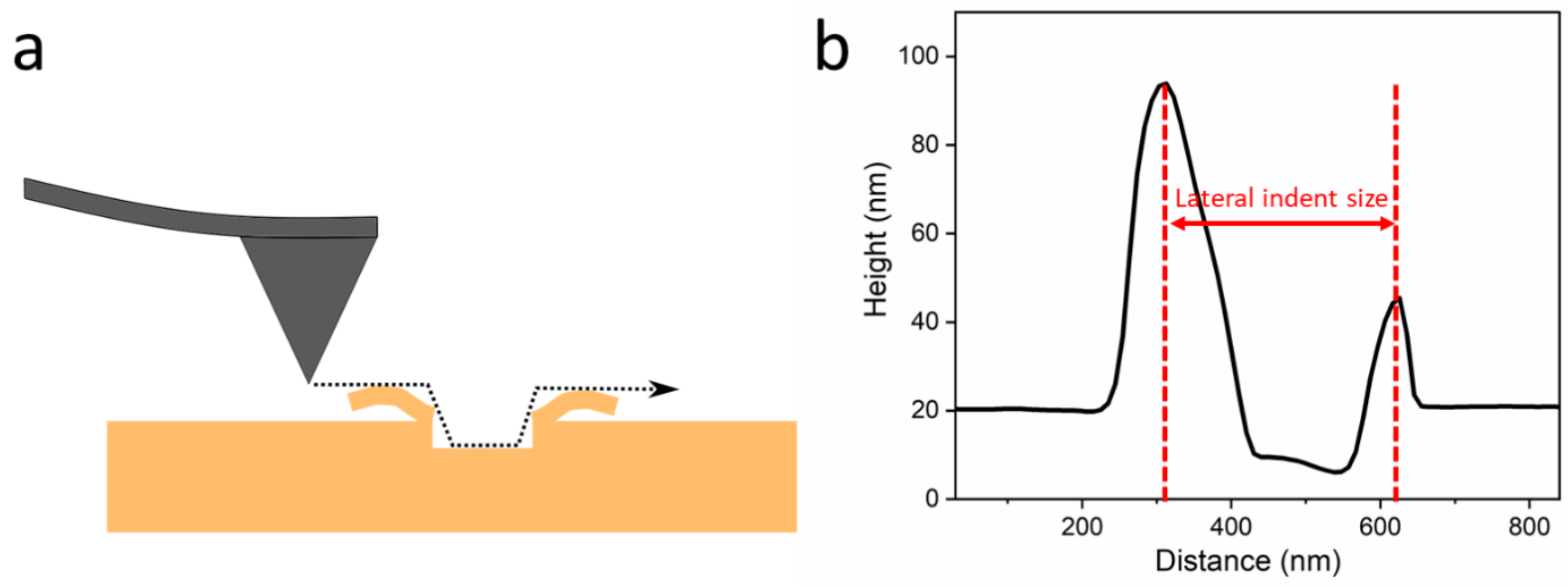

Figure S1. a) Schematic showing the effect of AFM cantilever when scanning an indent structure in hBN. Due to the width of the AFM tip, the indent profile cannot be accurately reproduced. b) Definition of the lateral indent size in this work, which refers to the distance between the two highest points across the indent. The height profile is taken from Figure 1d from the main text as an example.

\section{Dependence of the indentation depth on the maximum cantilever displacement}

Figure S2a shows an AFM image of a hBN flake after indentation. The indents were created with values of the maximum cantilever displacement along the vertical direction $\left(\Delta z_{\max }\right)$ indicated on the left of each indent row. The flake has a thickness of $\sim 11.5 \mathrm{~nm}$ as measured on the flake edge (Figure S2b). To show the indentation depth induced by different $\Delta z_{\max }$, the height profile across one representative indent was collected from each row of indents. As illustrated in Figure 
S2c, while the lateral indent size increases monotonically with $\Delta \mathrm{z}_{\max }$, the indentation depth shows little dependence on $\Delta z_{\max }$, with magnitudes comparable or less than the flake thickness for all $\Delta \mathrm{z}_{\max }$ values tested. Our results indicate that the indentation procedure used in this study has negligible effect or damage on the substrate underneath $\mathrm{hBN}$.
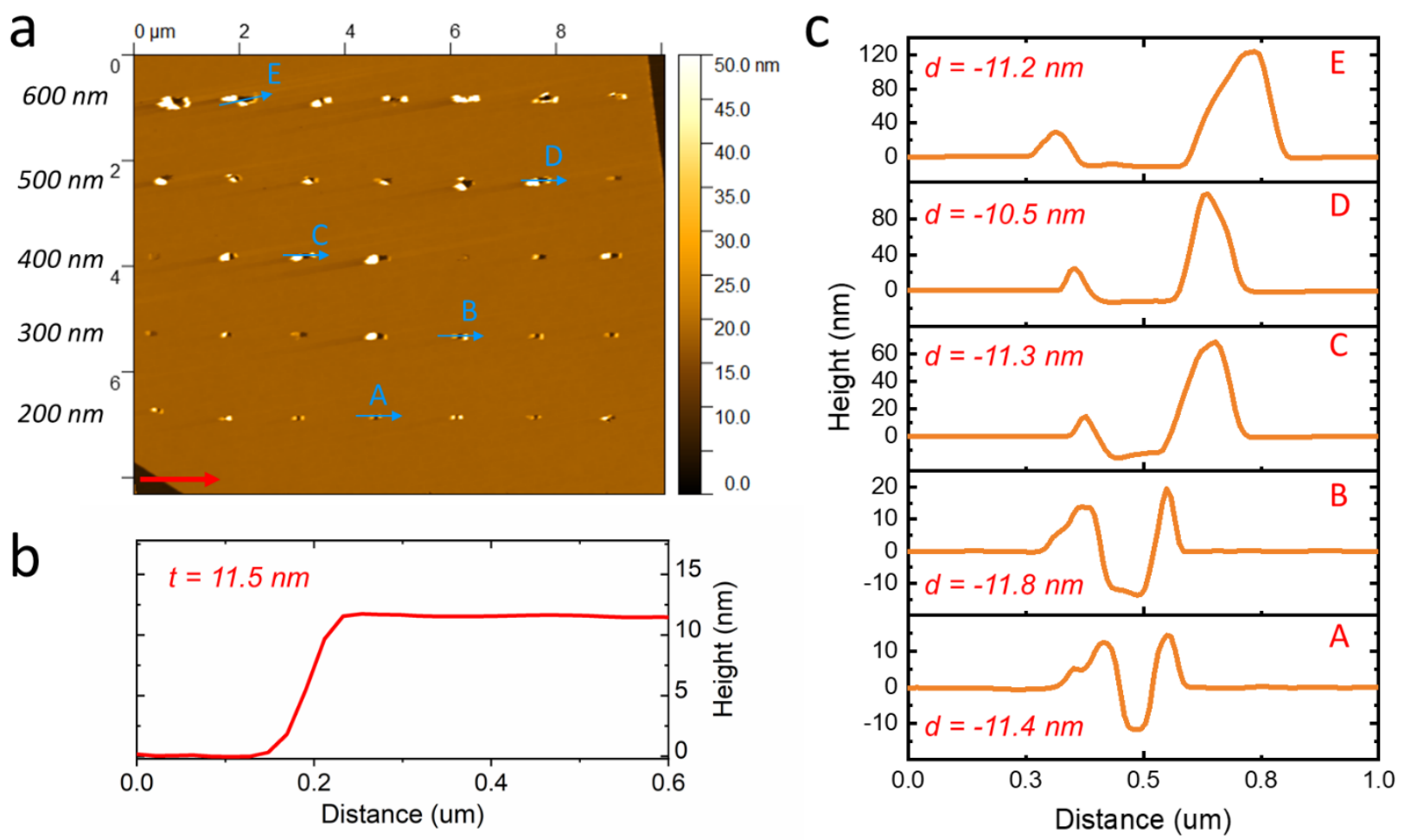

Figure S2. a) AFM image of an hBN flake after nanoindentation. Indents in the same row are created with the same maximum cantilever displacement $\Delta \mathrm{z}_{\max }$ as indicated on the left. b) Height profile along the solid red arrow in a), showing a flake thickness $t=11.5 \mathrm{~nm}$. c) Height profiles across the indents noted as A-E along the blue arrows in a). Value of $d$ noted for each indent in the plot indicates the measured indentation depth. 


\section{Additional data from hBN flakes used for optical measurement}
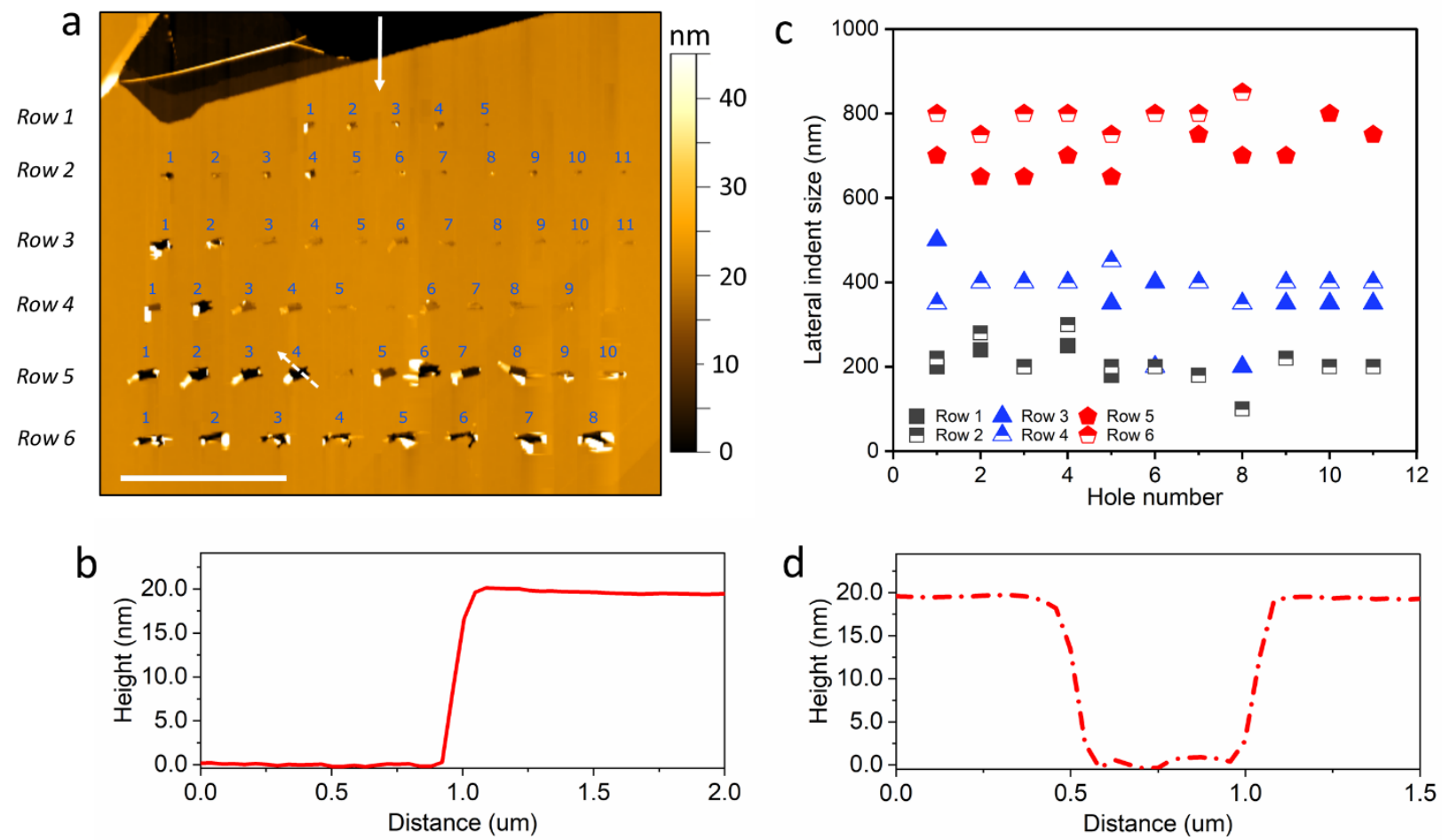

Figure S3. a) AFM image of the same flake shown in Figure 2a, with all indents numbered for reference. Scale bar: 5 um. b) Height profile at the flake edge along the solid white arrow in a), showing a flake thickness of $19.5 \mathrm{~nm}$. c) Distribution of the lateral indent size for all indents in a). Note: the indent sizes are measured along the lateral (horizontal) direction. $d$ ) The height profile of one of the largest indents (in terms of lateral size), i.e., indent number 4 in row 5, along the white dashed arrow, showing an indent depth comparable with the flake thickness. 

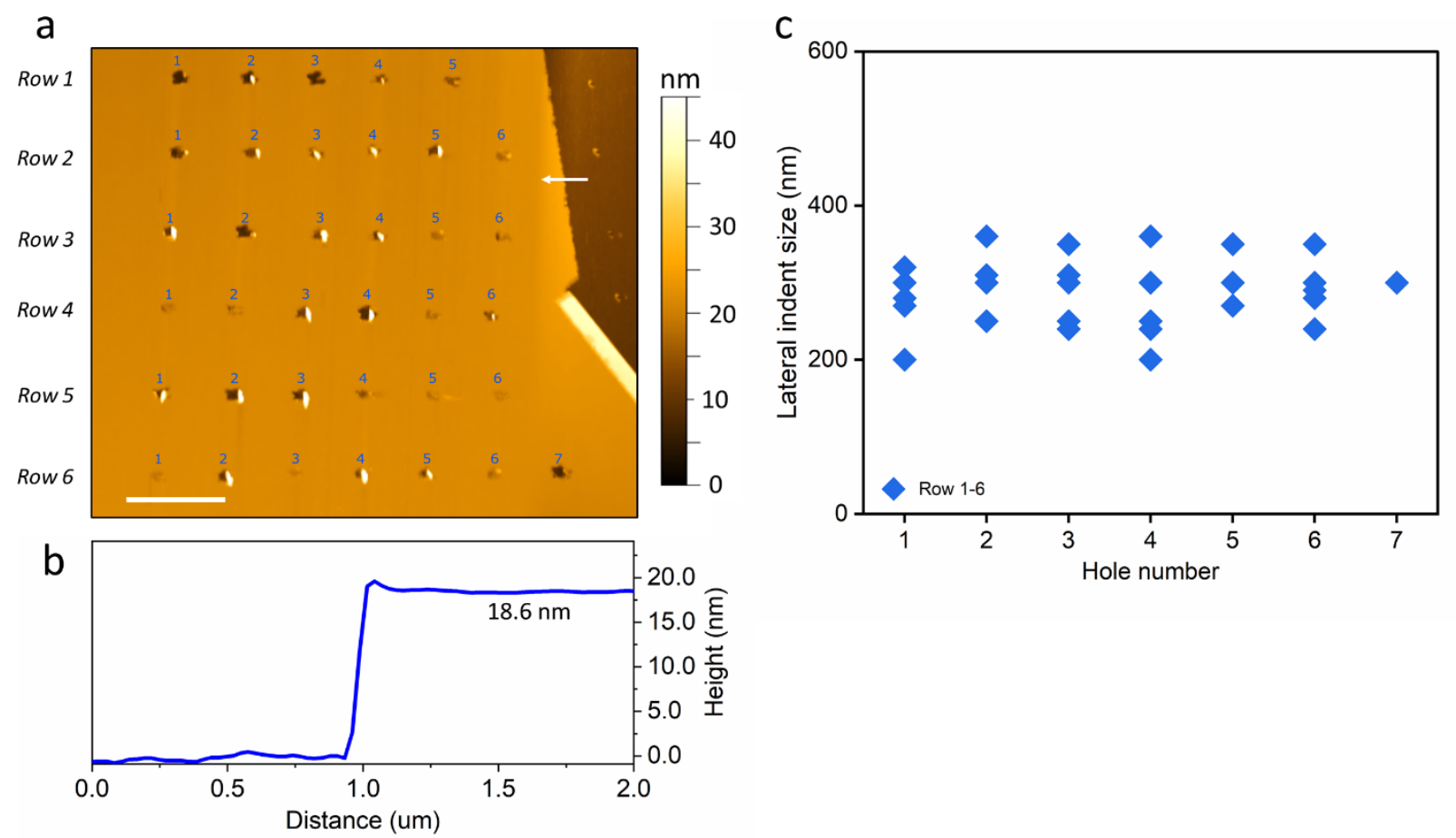

Figure S4. a) AFM image of the same flake shown in Figure 2d, with all indents numbered for reference. Scale bar: 2 um. b) Height profile at the flake edge along the solid white arrow in a), showing a flake thickness of $18.6 \mathrm{~nm}$. c) Distribution of the lateral indent size for all indents in a).

a

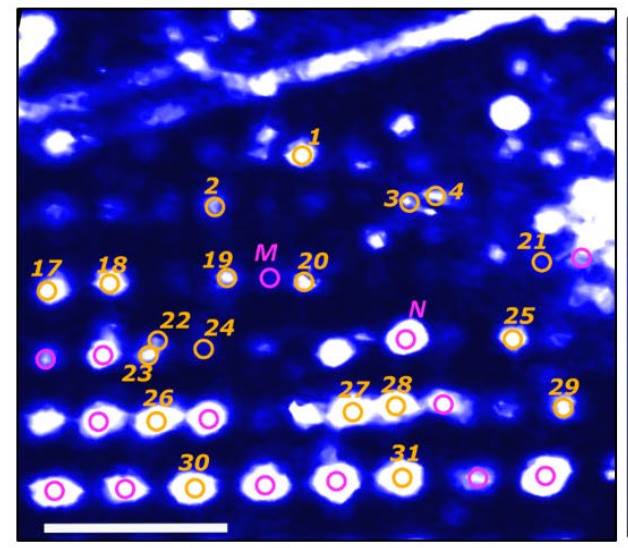

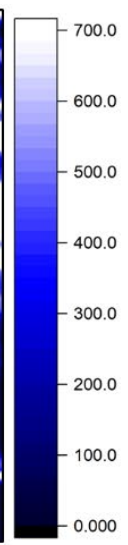

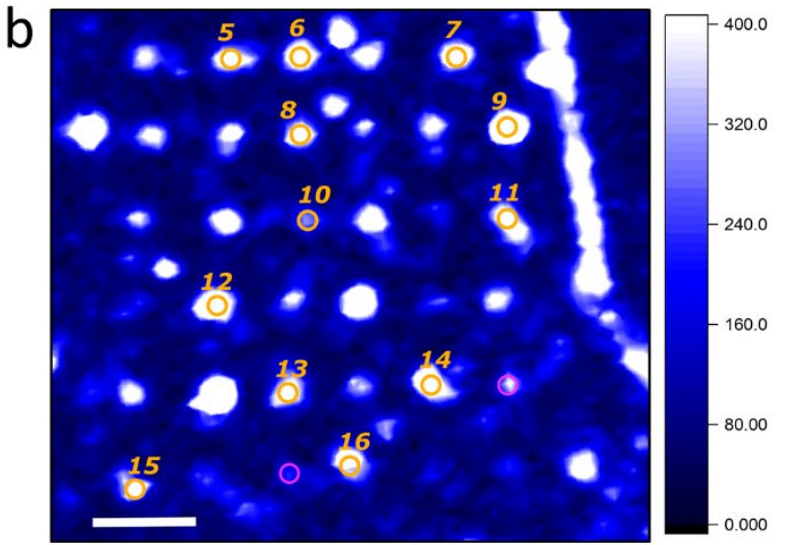

Figure S5. Notation of all emitters found on the two hBN flakes shown in Figure $2 \mathrm{~b} \&$ 2e. SPEs are circled and numbered in yellow, while clustered emitters are marked with magenta circles. 

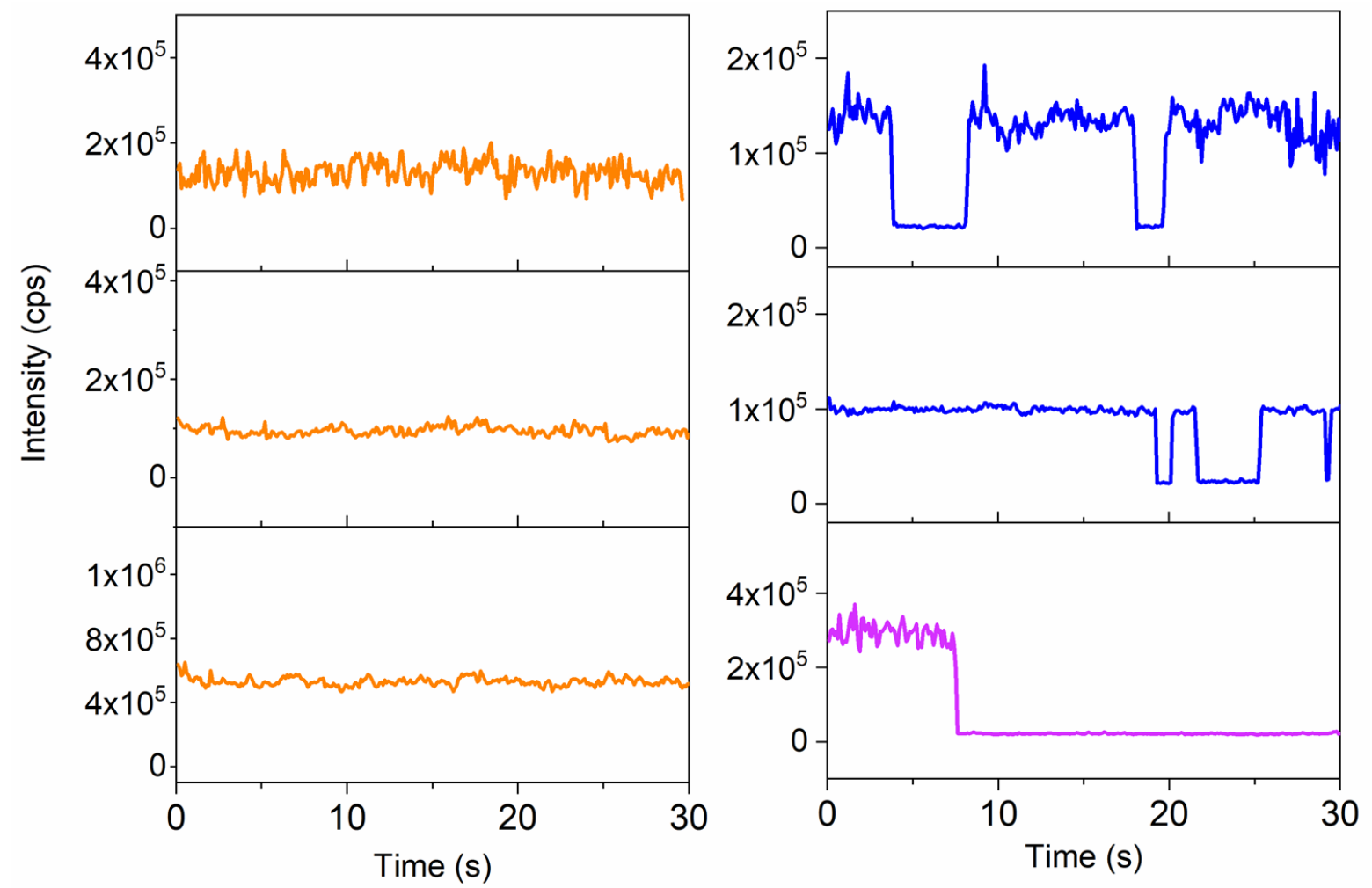

Figure S6. Stability of several representative SPEs showing photostable (three orange curves), blinking (two blue curves) and photobleached (one purple curve) emitters. 


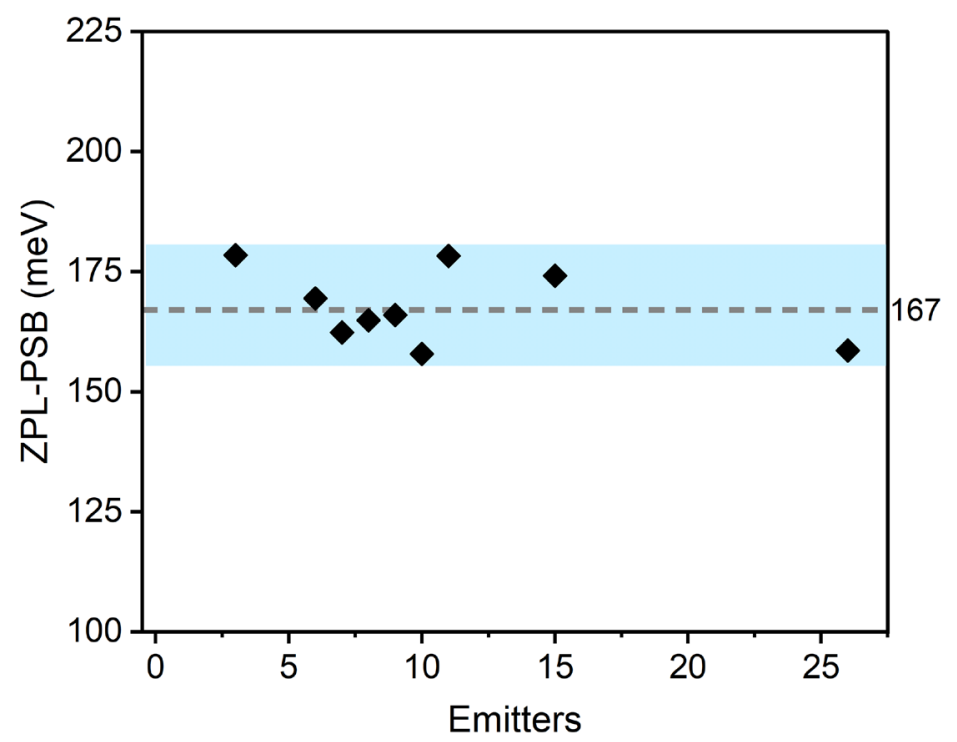

Figure S7. Difference in the energy of ZPL and PSB for SPEs that show well-defined PSBs in spectra. The numbering of emitters is consistent with Figure $3 \mathrm{~b}$ and Figure S4. The shaded band is a guide to the eye spanning over $167 \pm 10 \mathrm{meV}$. 

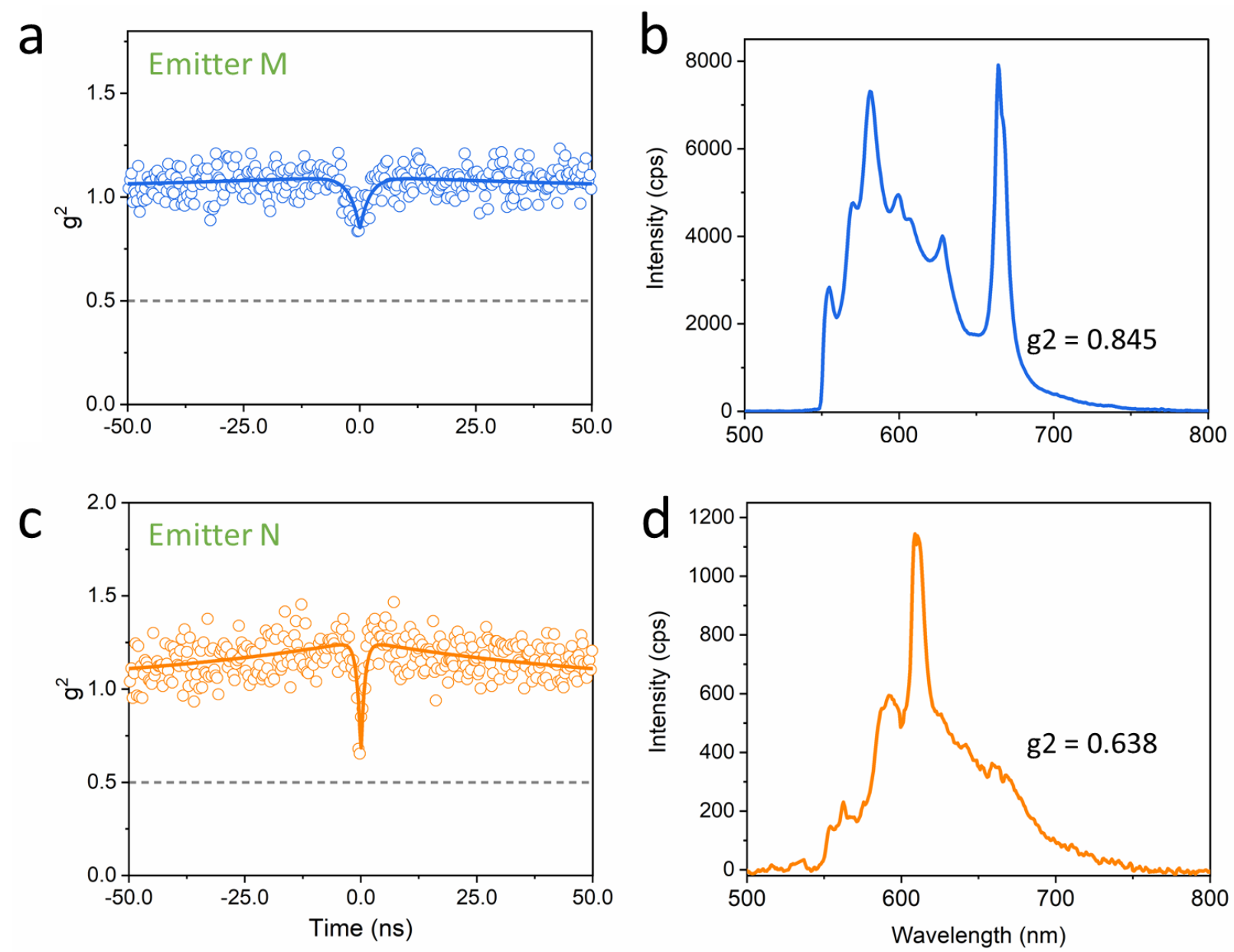

Figure S8. a) and c) Second-order autocorrelation measurements of two clustered emitters M \& N, both showing $0.5<\mathrm{g} 2(0)<1$. Locations of emitter $\mathrm{M} \& \mathrm{~N}$ are noted in Figure S4. c) and d) Corresponding spectra of Emitter $M \& N$ showing multiple emission peaks and broad emission, indicative of the existence of more than one SPEs per site.

\section{Control experiment with hBN flakes annealed at $800{ }^{\circ} \mathrm{C}$}

To investigate the effect of annealing temperature on the hBN emitter yield, we prepared additional hBN flakes, indented with AFM, and annealed them in argon at a lower temperature $\left(800^{\circ} \mathrm{C}\right)$ for 30 mins. Figure $\mathrm{S} 9 \mathrm{a}$ shows one hBN flake with arrays of indents that were all created with $\Delta \mathrm{z}_{\max }=350 \mathrm{~nm}$, the same $\Delta \mathrm{z}_{\max }$ that leads to a SPE yield of $32 \%$ at an annealing temperature of $1000{ }^{\circ} \mathrm{C}$. Here, the $\mathrm{hBN}$ flake has a thickness around $20 \mathrm{~nm}$ (Figure S9c) that is comparable to $\mathrm{hBN}$ flakes used in the main text. After argon annealing, the sample was again characterized with 
the confocal microscope to identify stable emitters. Figure S9b is a PL map of the indented area, with emitters identified in solid and dashed circles corresponding to SPEs and clustered emitters, respectively. SPEs found in this area share similar photophysical properties with those obtained by annealing at $1000{ }^{\circ} \mathrm{C}$ in terms of emission wavelength (S9d \& S9e). However, the SPE yield is calculated to be $12.8 \%$, much lower than that on hBN flakes annealed at $1000{ }^{\circ} \mathrm{C}(32 \%)$. Our observation above supports the hypothesis that atomic defects giving single photon emission at indented sites on hBN are vacancy-related structures: as the annealing temperature increases, the diffusion of vacancies is promoted, thus leading to a higher probability of forming vacancy-based defects that act as SPEs.
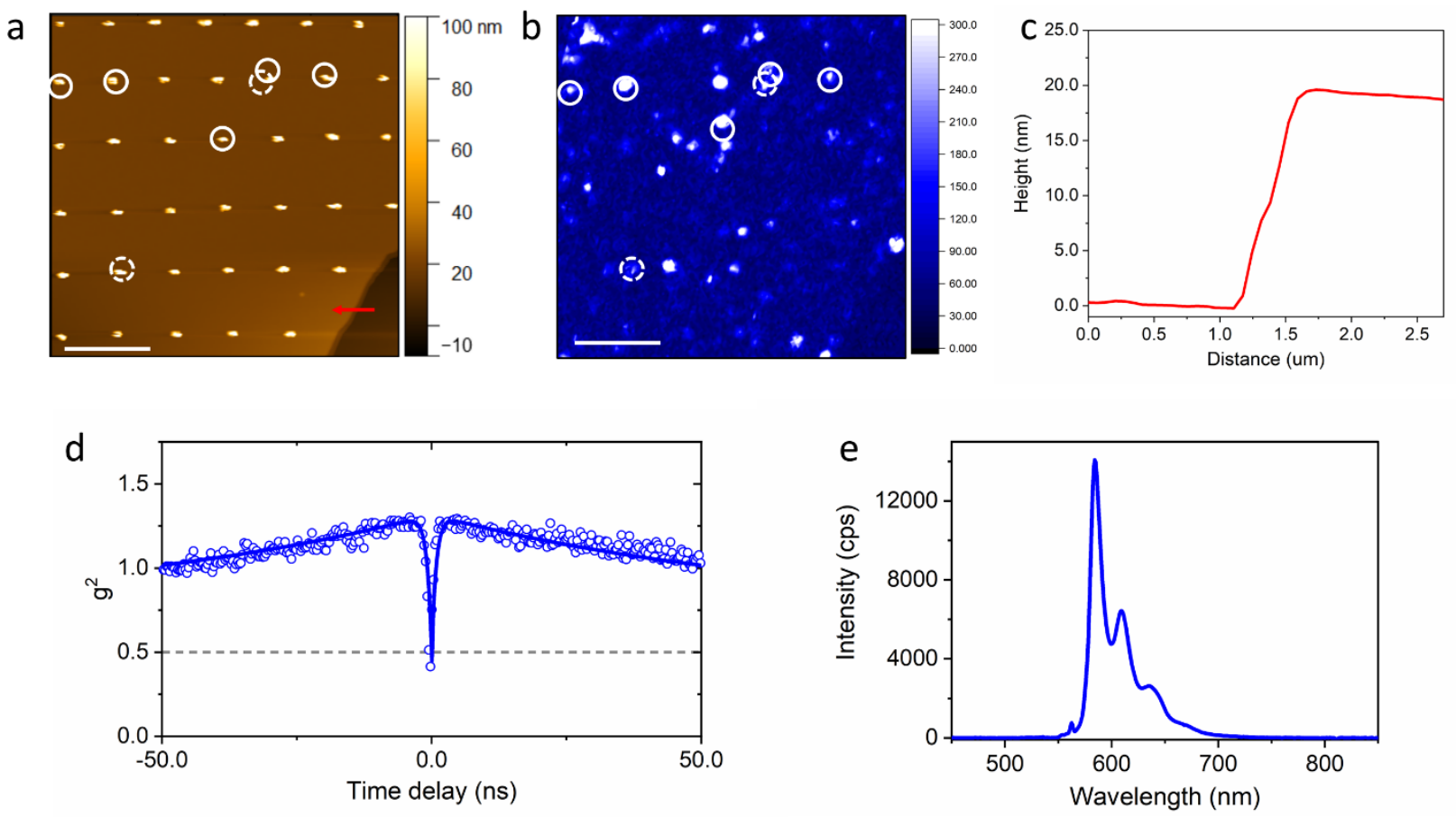

Figure S9. a) AFM image of an hBN flakes after nanoindentation. The flake was indented with the maximum cantilever displacement $\Delta \mathrm{z}_{\max }=350 \mathrm{~nm}$. Scale bar: $2 \mathrm{um}$. b) PL maps of the same indented areas shown in a) after being annealed in argon at $800^{\circ} \mathrm{C}$. SPEs and clustered emitters are found and marked with while solid and dashed circles, respectively. Scale bar: $2 \mathrm{um}$. The corresponding indents are also circled out in a). c) Height profile at the flake edge along the red arrow marked in a), showing a flake thickness of $\sim 20.0 \mathrm{~nm}$. d) Second-order autocorrelation measurement and fitting of a SPE from the area shown in a), The blue circles are experimental data, while the solid blue line is the fitting curve with the three-level model. e) Background-subtracted spectrum of the same SPE, showing a ZPL at $581 \mathrm{~nm}$ and a PSB at the longer wavelength. 


\section{References}

(1) Braga, P. C.; Ricci, D. Atomic Force Microscopy: Biomedical Methods and Applications; 2004.

(2) Keller, D. Reconstruction of STM and AFM Images Distorted by Finite-Size Tips. Surf. Sci. 1991, 253, 353-364. 